\title{
Unsteady Vorticity Shedding from a Circular Cylinder: Surging, Spinning and Gust Encounters
}

\author{
P. Gehlert* and H. Babinsky ${ }^{\dagger}$ \\ University of Cambridge, Cambridge, United Kingdom, CB2 1PZ
}

\begin{abstract}
The unsteady shedding process of circulation from a translating and rotating cylinder is investigated using planar particle image velocimetry, both in quiescent fluid as well as during a sharp-edged gust encounter. The Reynolds numbers are 20000 and 6000 , respectively. The rate at which circulation is shed is computed by evaluating the vorticity flux in the boundary layer. The shedding rate is found to be proportional to the difference between the total boundary layer vortex sheet and the vortex sheet strength contribution due to rotation. This is equivalent to the sum of the non-circulatory vortex sheet and that due to shed circulation, evaluated at the separation point. A direct consequence of this is that the shedding rate is independent of body rotation. It only depends on the geometry of the body and any previously shed circulation. Therefore, before any separation has occurred and if the separation point is known, the rate at which circulation sheds can solely be determined from potential flow theory. It is also observed that previously shed circulation, which has travelled a large distance from the cylinder, induces a uniform 'mirror' vortex sheet, which has the effect of globally affecting and shifting the boundary layer vortex sheet. This can potentially provide an alternative explanation for alternate vortex shedding. Similarly, Prandtl's theoretical maximum lift coefficient for a surging and rotating cylinder can be reconciled with the proposed theory and recovered by only using this boundary layer vortex sheet analysis.
\end{abstract}

\section{Selected Nomenclature}

\begin{tabular}{llll}
$\alpha$ & Rotation ratio & $\theta$ & Angle definition along cylinder (rad) \\
$\delta$ & Boundary layer height $(\mathrm{m})$ & $a$ & Cylinder radius (m) \\
$\dot{\Gamma}_{b l}$ & Vorticity flux in the boundary layer $\left(\mathrm{m}^{2} / \mathrm{s}^{2}\right)$ & $D$ & Cylinder diameter $(\mathrm{m})$ \\
$\dot{\Gamma}_{s h e d}$ & Rate at which circulation is shed $\left(\mathrm{m}^{2} / \mathrm{s}^{2}\right)$ & $G R$ & Gust Ratio \\
$\Gamma$ & Circulation $\left(\mathrm{m}^{2} / \mathrm{s}\right)$ & $s$ & Cylinder position (m) \\
$\gamma$ & Vortex sheet strength $(\mathrm{m} / \mathrm{s})$ & $s_{\Omega}$ & Distance in which cylinder reaches terminal \\
$\gamma_{b}$ & Boundary layer vortex sheet $(\mathrm{m} / \mathrm{s})$ & $s_{a c c}$ & Distance in which cylinder accelerates (m) \\
$\gamma_{c}$ & Vortex sheet due to shed vorticity $(\mathrm{m} / \mathrm{s})$ & $U$ & Instantaneous streamwise velocity (m/s) \\
$\gamma_{n c}^{a m}$ & Vortex sheet from translation / added mass $(\mathrm{m} / \mathrm{s})$ & $U_{\infty}$ & Steady-state streamwise velocity (m/s) \\
$\gamma_{n c}^{e x t}$ & Vortex sheet due to external gust vorticity $(\mathrm{m} / \mathrm{s})$ & $U_{e}$ & Boundary layer edge velocity (m/s) \\
$\gamma_{r}$ & Vortex sheet due to rotation $(\mathrm{m} / \mathrm{s})$ & $U_{w}$ & Wall velocity (m/s) \\
$\Omega$ & Angular velocity (rad/s) & $U_{b l}$ & Boundary layer velocity (m/s) \\
$\omega$ & Vorticity (1/s) & $V$ & Instantaneous vertical gust velocity (m/s) \\
$\bar{V}$ & Average vertical gust velocity (m/s) & $v$ & Vertical gust velocity along gust width (m/s) \\
\hline
\end{tabular}




\section{Introduction}

$\mathrm{R}$ APIDLY changing flow fields can result in dramatically fluctuating force responses. Many of the common assumptions, such as small angles of attack or small velocity perturbations can no longer be applied. Initially, added mass effects dominate. As time passes, vorticity forms on the body surface and begins to shed. The growth and advection of the vortices, subsequently strongly affects the overall forces [1]. Consequently, in order to quickly compute the expected forces, numerous investigations have sought to better understand this unsteady flow field and to develop low order models (LOM) [2-4]. A successful implementation of these would allow for gust rejection systems, for example for micro aerial vehicles that are often compromised by high gust rations and turbulent fluctuations occurring in their flight envelopes [5-7].

Crucial to this endeavour is the correct prediction of the rate at which circulation is shed into the flow. It is hypothesised that the shed circulation is linked to the surface vortex sheet at the point of separation. This implies that whilst the flow is fully attached, the amount of circulation shed can be predicted exactly from potential flow. The boundary layer vortex sheet is equal to the slip velocity between the body surface and the flow. When no circulation has been shed, the flow velocity is affected only by the potential flow field resulting from the body geometry and the respective kinematics. Shed circulation does not exist and hence, does not influence the slip velocity. If the location where separation first occurs is known a priori, then from the surface vortex sheet, the exact shedding rate of circulation can be calculated.

The aim of this paper is therefore, to shed light on this unsteady shedding behaviour. The primary objective is to determine whether it is possible to evaluate how much circulation is shed at a given instance in time by only evaluating the boundary layer vorticity. Specifically, the effect of body rotation and any previously shed circulation is assessed.

The study is conducted using a surging and rotating circular cylinder and allows for a thorough inspection of the underlying fundamental principles. While previous investigations of unsteady flows have often utilised flat plates or aerofoils, a circular cylinder has the advantage that it includes volume effects, does not fix the separation location and, by varying the spin rate, enables an assessment of different lift coefficients without requiring a change in geometry. A sharp-edged gust encounter of the same cylinder extends the results to a more complex flow field, testing any previous assumptions.

\section{Predicting Shedding Rate by Evaluating Vorticity Flux}

To evaluate the rate at which circulation is shed into the flow field, the boundary layer is represented by a vortex sheet [8]. The total boundary layer vortex sheet, $\gamma_{b}$, has a number of sources that contribute to its absolute strength [9-14] such that

$$
\gamma_{b}=\gamma_{n c}^{a m}+\gamma_{n c}^{e x t}+\gamma_{c}+\gamma_{r} .
$$

$\gamma_{n c}^{a m}$ is derived from enforcing the no penetration condition due to the translational motion of the body and is therefore, related to the added mass effect. $\gamma_{n c}^{\text {ext }}$ is the result of circulation not shed by the body but created through external means, for example a gust shear layer. The related circulation induces velocities on the body surface that requires $\gamma_{n c}^{e x t}$ to maintain the no through flow condition. Similarly, any circulation shed by the body also induces velocities on the body surface. Consequently, after separation has occurred a further vortex sheet, $\gamma_{c}$, is required to guarantee no through flow. Finally, $\gamma_{r}$ exists because of the slip velocity between a rotating body and surrounding quiescent fluid. The two non-circulatory vortex sheets $\gamma_{n c}^{a m}$ and $\gamma_{n c}^{e x t}$ will from now on be referred to as $\gamma_{n c}$ to remove any undue clutter in the equations. For the present cases, it is assumed that they can be fully determined from the kinematics or a knowledge of the externally enforced vertical gust strength and location. When exploring the surging and rotating cylinder in a quiescent fluid, $\gamma_{n c}$ is equal to $\gamma_{n c}^{a m}$, as $\gamma_{n c}^{e x t}$ is zero due to the absence of any externally created circulation.

The rate at which vorticity is transported along in the boundary layer, the vorticity flux, can be expressed as

$$
\dot{\Gamma}_{b l}=\frac{d \Gamma}{d t}=\int_{\delta} \omega u d n,
$$

where $\delta$ is the boundary layer thickness. $\omega$ and $u$ represent the vorticity and velocity distribution in the wall normal $n$ direction [15]. A schematic illustration of this is shown in figure 1 


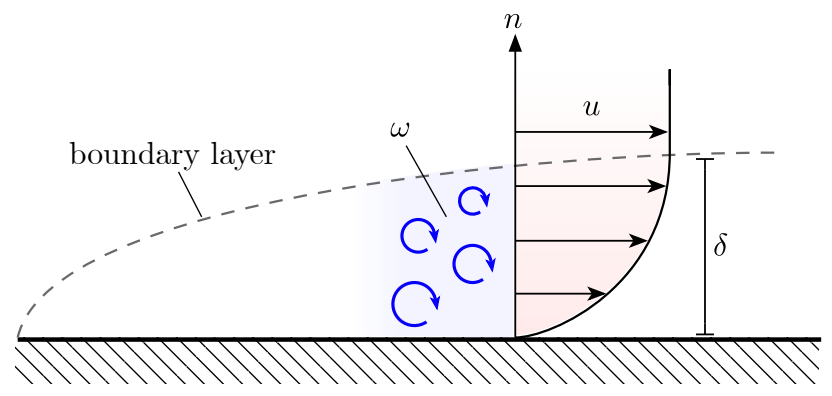

Fig. 1 Calculating $\dot{\Gamma}_{b l}$ by integrating the velocity, $u$, and vorticity, $\omega$, distribution.

Using the definition of the boundary layer vortex sheet, the vorticity flux can be re-written as

$$
\dot{\Gamma}_{b l}=\gamma_{b} U_{b l},
$$

where $U_{b l}$ is the average velocity inside the boundary layer. At the point of separation, the circulation is not transported to the next position along the cylinder but into the flow field and the amount of shed circulation can therefore be determined. Consequently, the vorticity flux is directly related to the amount of circulation shed. Figure 2 schematically demonstrates this idea.

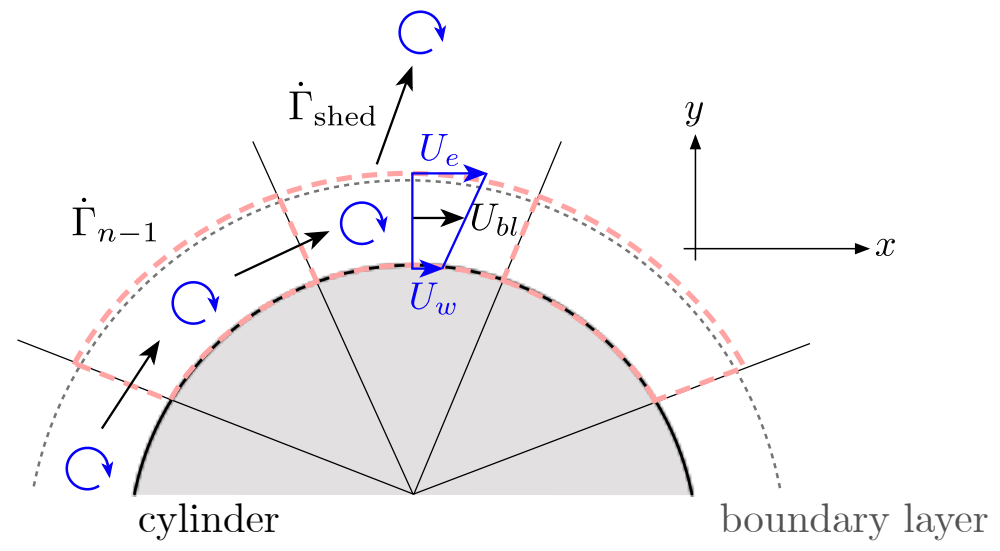

Fig. 2 Schematic illustration of the vorticity flux at the point of separation being transported into the flow field.

By investigating how the contributions to $\gamma_{b}$ and $U_{b l}$ change in time, an understanding of why the rate of vortex shedding varies can be obtained. From equation 1, $\gamma_{b}$ is a function of the individual vortex sheet components,

$$
\gamma_{b}=f\left(\gamma_{n c}, \gamma_{c}, \gamma_{r}\right)
$$

The boundary layer velocity, as a first order approximation, is assumed to be the average between the velocity of the flow at at edge of the boundary layer, $U_{e}$ and that at the wall, $U_{w}$,

$$
U_{b l}=\frac{U_{w}+U_{e}}{2}
$$

Ideally, equation 3 is expressed solely in terms of vortex sheet components, to provide a consistent approach to identifying contributions that cause $\dot{\Gamma}_{b l}$ to vary. The following derivation is applied to a point on the lower half of the cylinder but can equally be repeated for a postion on the upper surface.

Expressing the wall velocity $U_{w}$ in terms of vortex sheet components links it to the rotational contribution:

$$
U_{w}=-\gamma_{r}
$$


The boundary layer edge velocity $U_{e}$ is affected by the potential flow due to translation and externally created circulation, $\gamma_{n c}$ as well as by any shed circulation, $\gamma_{c}$. Since $\gamma_{b}=\gamma_{n c}+\gamma_{c}+\gamma_{r}, U_{e}$ is expressed as

$$
U_{e}=\gamma_{b}-\gamma_{r}
$$

Substituting both equation 6 and 7 into equation 5 leads to

$$
U_{b l}=\frac{\gamma_{b}-2 \gamma_{r}}{2}
$$

Inserting equation 8 into the initial equation for $\dot{\Gamma}_{b l}$, equation 3 , yields

$$
\dot{\Gamma}=\frac{\gamma_{b}-2 \gamma_{r}}{2} \gamma_{b}
$$

which is only a function of $\gamma_{n c}, \gamma_{c}$ and $\gamma_{r}$. Therefore, resulting in the vorticity flux being governed only by the vortex sheet components,

$$
\dot{\Gamma}=f\left[\gamma_{b}\left(\gamma_{n c}, \gamma_{c}, \gamma_{r}\right)\right]
$$

By definition, the velocity at the point of separation is entirely normal to the surface. Therefore, there exists no tangential velocity component that contributes to the surface vortex sheet. Only the slip velocity between the rotating cylinder and nominally quiescent fluid affects $\gamma_{b}$. Consequently, $\gamma_{b}$ is equal to $\gamma_{r}$ at the point of separation. Vorticity and the boundary layer velocity distribution, just before and after the separation point, are schematically illustrated in figure 3 .

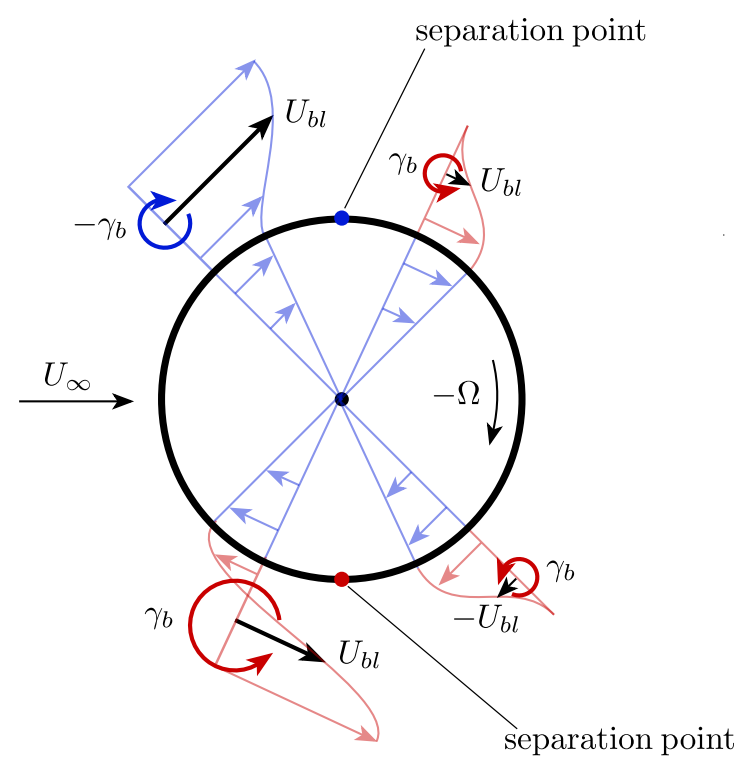

Fig. 3 Schematic illustration of vorticity flux and the velocity profile inside and outside a spinning cylinder in uniform freestream.

Assuming that the vorticity flux is conserved at the separation point, implies that the difference between the vorticity flux just prior to separation and just after must equal the amount of circulation shed. Hence, the rate at which circulation is shed can be expressed as:

$$
\dot{\Gamma}_{\text {shed }}=\left.\gamma_{b} U_{b l}\right|_{\text {before }}-\left.\gamma_{b} U_{b l}\right|_{\text {after }}=\left.\gamma_{b} \frac{\gamma_{b}-2 \gamma_{r}}{2}\right|_{\text {before }}-\left.\gamma_{b} \frac{\gamma_{b}-2 \gamma_{r}}{2}\right|_{\text {after }}
$$

Replacing $\gamma_{b}$ by its respective vortex sheet components and letting $\gamma_{b}=\gamma_{r}$ just after separation, leads to

$$
\dot{\Gamma}_{\text {shed }}=\frac{\left(\gamma_{n c}^{\text {sep. }}+\gamma_{c}^{\text {sep. }}\right)^{2}}{2}=\frac{\left(\gamma_{b}^{\text {sep. }}-\gamma_{r}^{\text {sep. }}\right)^{2}}{2} \text {. }
$$




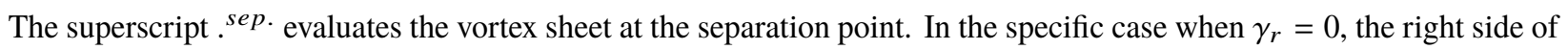
equation 12 is similar to the formulations derived by, amongst others, Saffman \& Schatzman [8], Sarpkaya \& Schoaff [16] and Xia \& Mohseni [17]. Schematically, the result of equation 12 is visualised in figure 4 . The amount of circulation

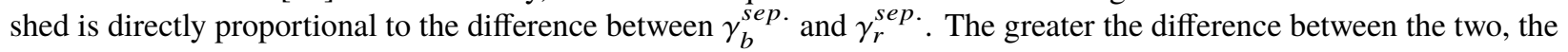
more circulation is shed.

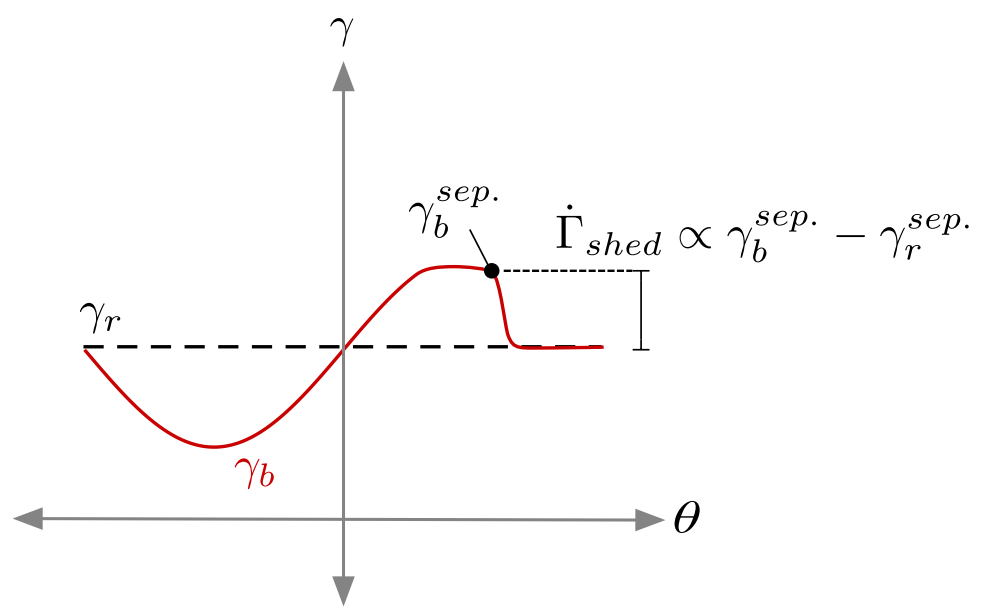

Fig. 4 Schematic illustration of the surface vortex sheets and the dependency of $\dot{\Gamma}_{\text {shed }} ; \dot{\Gamma}_{\text {shed }} \propto \gamma_{b}-\gamma_{r}$

The preceding derivation can equally be repeated for a point on the upper half of the cylinder, as long as the sign convention is applied rigorously. Defining $U_{b l}$ in terms of vortex sheet components, requires careful attention to the sign of the vortex sheets, as a positive velocity in the general coordinate system (positive = left to right) will require a change in sign when moving from the upper portion of the cylinder to the bottom half. For example $\gamma_{r}$ will be identical on both sides of the cylinder but the surface of a clockwise spinning cylinder will be travelling from left to right on the upper half and from right to left on the bottom side. A schematic illustration of this for rotating cylinder in quiescent fluid is shown in figure 5 .

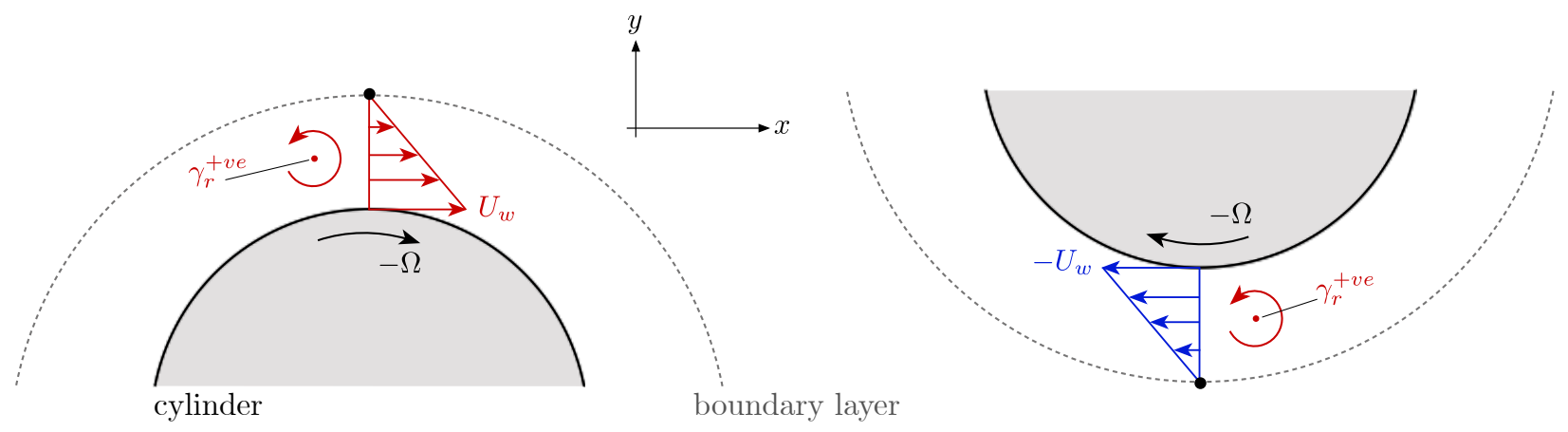

Fig. $5 \gamma_{r}$ stays constant but $U_{w}$ changes sign when moving from the upper to the lower half of the cylinder; flow field represents a rotating cylinder in quiescent fluid.

Alternatively, the first formulation of $\dot{\Gamma}_{\text {shed }}$ in equation 12 implies that

$$
\dot{\Gamma}_{\text {shed }}=f\left(\gamma_{n c}^{\text {sep. }}, \gamma_{c}^{\text {sep. }}\right)
$$

Whilst the vorticity flux inside the boundary layer is dependent on the rotation rate, the rate at which circulation is shed does not have an explicit dependence on it. Of course however, there is still an implicit contribution from the rotation 
rate, as it will affect the location of the separation point and the time history of shed circulation. The independence on rotation rate might be a surprising result and Goldstein [18] agreed that the rotation rate should affect the amount of shed circulation, as the strength of the vortex sheet is strongly affected by the rotation rate. This is because rotation causes the wall to move with or against the flow, hence changing the relative velocity. However, the vorticity flux is also affected by how quickly this vorticity is transported. The upper surface, of a clockwise spinning cylinder in uniform freestream from left to right, creates less boundary layer vorticity than its bottom counterpart but the advection velocity is higher. Furthermore, the amount of circulation shed is directly proportional to the boundary layer velocity. This is a direct consequence of equation 12, as $\gamma_{n c}^{\text {sep. }}$ scales with instantaneous velocity. Manar et al. demonstrated this for a surging flat plate at incidence [19].

The sole dependency of $\dot{\Gamma}_{b l}$ on boundary layer vortex sheet components is powerful, as it directly links the boundary layer strength at the point of separation, $\gamma_{b}^{\text {sep. }}$, to the rate at which circulation is shed. By understanding which flow components cause $\gamma_{b}^{\text {sep. }}$ to change, it is possible to infer why a corresponding variation in shedding rate is observed.

\section{Experimental Investigation into Cylinder Vortex Shedding}

To explore the unsteady shedding process and to investigate the proposed ideas, a circular cylinder is subjected to surge and rotation. More specifically, the first experimental set-up studies the flow development behind a surging or surging and rotating cylinder in quiescent fluid, whilst the second investigates the flow field when the same cylinder enters a top-hat shaped gust.

\section{A. Experimental Set-Up}

\section{Test Geometry and Kinematics}

The experiments are performed in the towing tank facilities of the University of Cambridge. The $1 \mathrm{~m}$ wide and $9 \mathrm{~m}$ long water tank is filled to a depth of $0.8 \mathrm{~m}$ for all test cases. A $0.48 \mathrm{~m}$ long cylinder with a diameter of $0.06 \mathrm{~m}$ is vertically mounted to an electronically communicated (EC) motor, which is positioned on a force balance connected to a carriage. This allows simultaneous rotation and translation. An illustration of the test cases is shown in figure 6 .

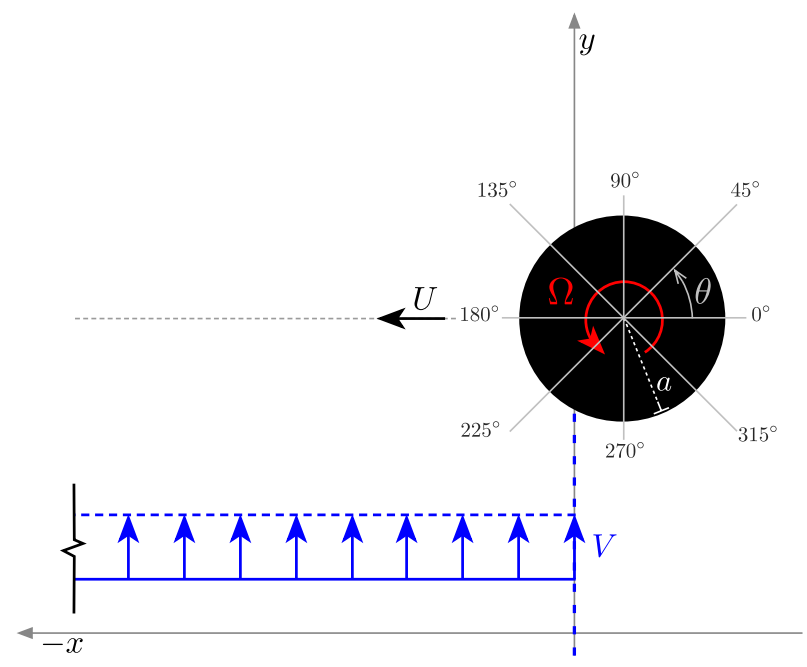

Fig. 6 Experimental configuration. The vertical gust velocity is zero when the surge and rotation experiments are performed in quiescent fluid. The angle definition, $\theta$, around the cylinder is also indicated, as is the radius, $a$.

Schematic illustrations of the test arrangements for both the surge and rotation cases as well as the cylinder gust encounter are shown in figures 7 and 8 . A more detailed description of the cylinder set-up can be found in [12]. 


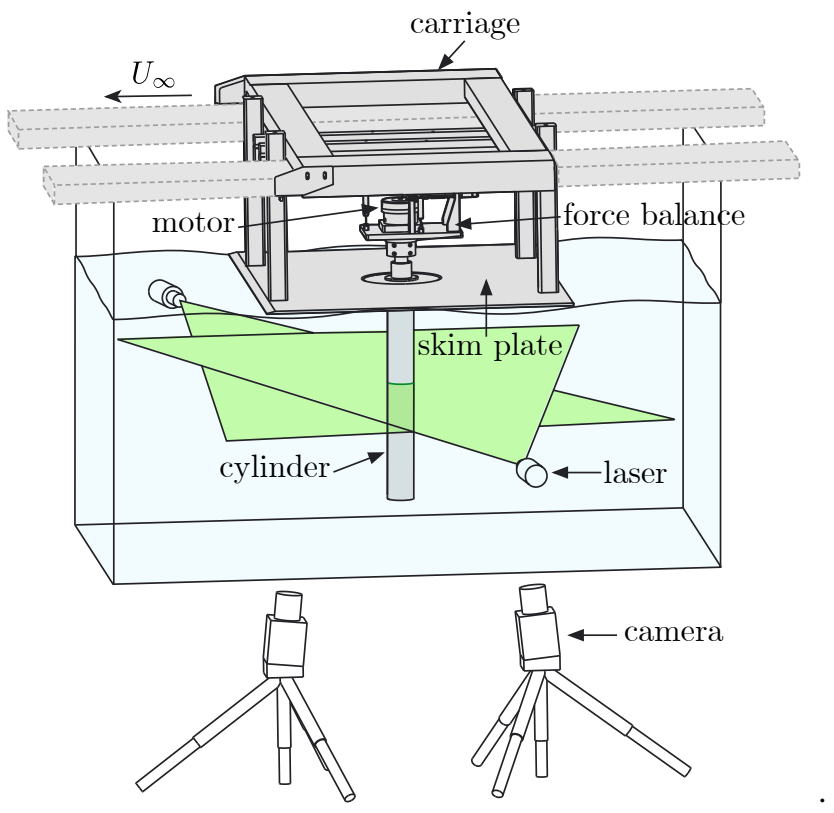

Fig. 7 Experimental set-up of the surging and rotating cylinder.

Case 1 - Surging and Rotating Cylinder

The cylinder begins to translate from a stationary start. It accelerates over a distance of two to three diameters after which it continues to translate at a constant velocity of $0.43 \mathrm{~ms}^{-1}$. This gives a steady state Reynolds number, $R e$, of $2 \times 10^{4}$. When the cylinder surges and rotates, it begins both motions simultaneously. A rotation ratio, $\alpha=\frac{\Omega_{\infty} a}{U_{\infty}}$, of 1 is achieved with a spin rate of 137 revolutions per minute (RPM). A constant angular velocity, $\Omega_{\infty}$, is reached within $s / D=0.15$.

\section{Case 2 - Cylinder Gust Encounter}

The cylinder begins its surging motion $1.24 \mathrm{D}$ upstream of the gust from a stationary start. It accelerates within $0.5 \mathrm{D}$ to a final velocity of $0.129 \mathrm{~ms}^{-1}$. The Reynolds number, based on final cylinder surge velocity, is 6000 . $R e$ is reduced because the velocity of the transverse gust is limited by the power of the pump. Additionally, the measured particle image velocimetry data is cleaner for lower $R e$ as the flow in the wake is less three-dimensional and the repeatability is also improved. The force coefficients and the flow field for a surging and rotating cylinder in quiescent fluid were found to be invariant to Reynolds number changes in this regime and gust experiments on a flat plate showed similar results [20]. The cylinder rotates at a rotation ratio of 1 and the gust ratio, $G R$ is 1.

A summary of the kinematic parameters is provided in table 1 .

\begin{tabular}{cccccccc}
\hline \hline & $\boldsymbol{\alpha}$ & $\boldsymbol{R P M}$ & $\boldsymbol{U}_{\infty}\left(\mathrm{ms}^{-\mathbf{1}}\right)$ & $\boldsymbol{R e}$ & $\boldsymbol{G R}$ & $\boldsymbol{s}_{\mathrm{acc}} / \boldsymbol{D}$ & $\boldsymbol{s}_{\boldsymbol{\Omega}_{\infty}} / D$ \\
\hline Case 1 & 0 & 0 & 0.43 & $2 \times 10^{4}$ & - & 3 & - \\
\hline Case 2 & 1 & 137 & 0.43 & $2 \times 10^{4}$ & - & 2 & 0.15 \\
\hline Case 3 & 1 & 41 & 0.129 & $6 \times 10^{3}$ & 1 & 0.5 & 0.04 \\
\hline \hline
\end{tabular}

Table 1 Summary of the test cases. $s_{\text {acc }}$ represents the acceleration distance and $s_{\Omega_{\infty}}$ the distance required to reach terminal angular velocity, $\Omega_{\infty}$. 


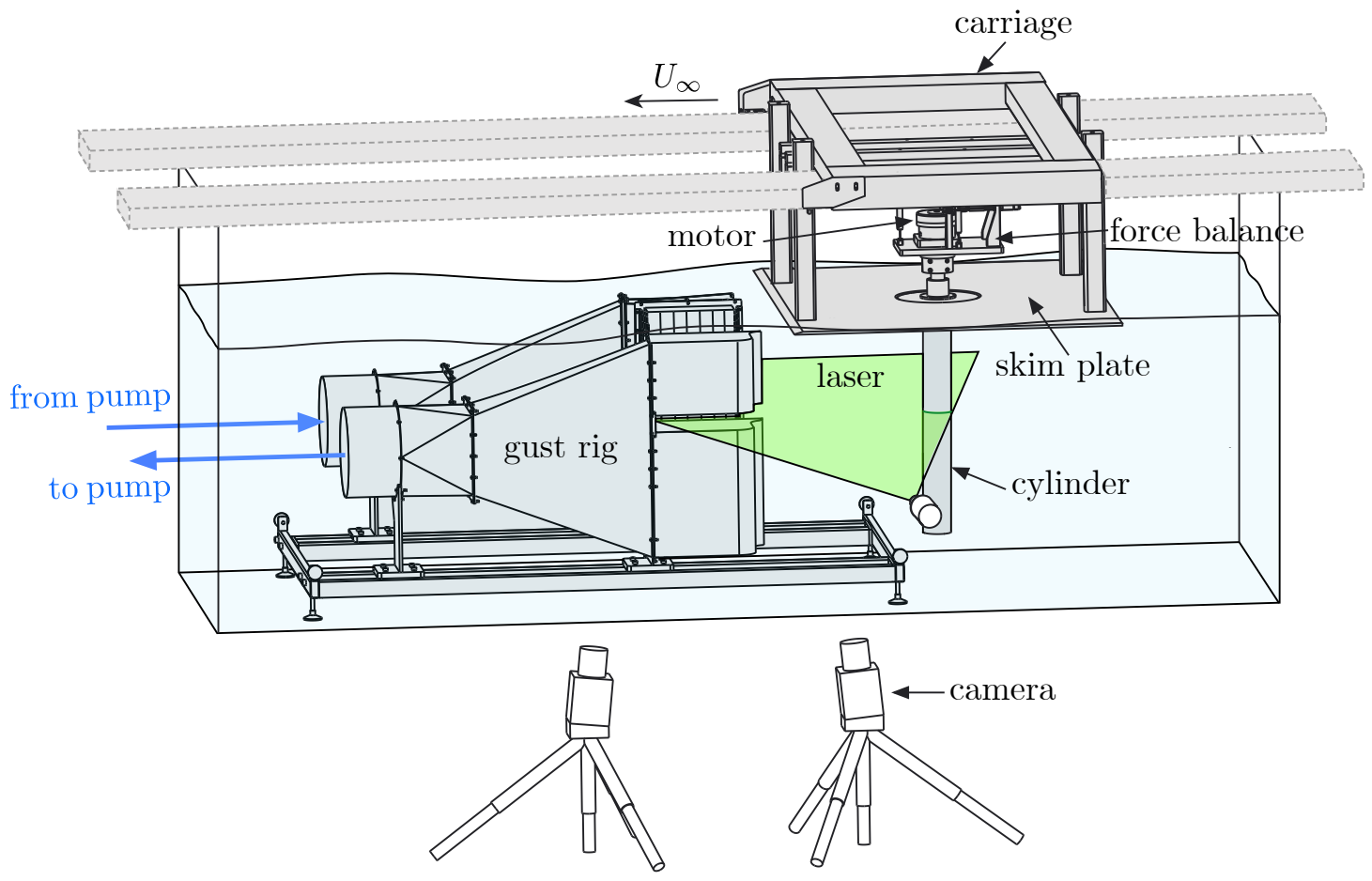

Fig. 8 Experimental set-up including the gust rig .

The gust rig [21] generates a sharp-edged top-hat velocity profile, as shown in figure 9 . The cylinder centre is offset by $0.1 \mathrm{~m}$ or $\frac{3}{5} D$ from the gust outlet. The path taken by the cylinder is indicated in figure 9a together with the vorticity field. The vertical gust velocities at three locations inside the gust are shown in figure $9 \mathrm{~b}$ The gust shear layers diffuse further away from the gust outlet, leading to a less sharp gust profile. Furthermore, a small yet non-zero velocity is recorded outside the gust. This is due to flow being entrained by the gust, however, its magnitude is negligible compared to the gust velocity itself. A more complete description of the gust rig and the respective cylinder gust encounter can be found in [14, 21].

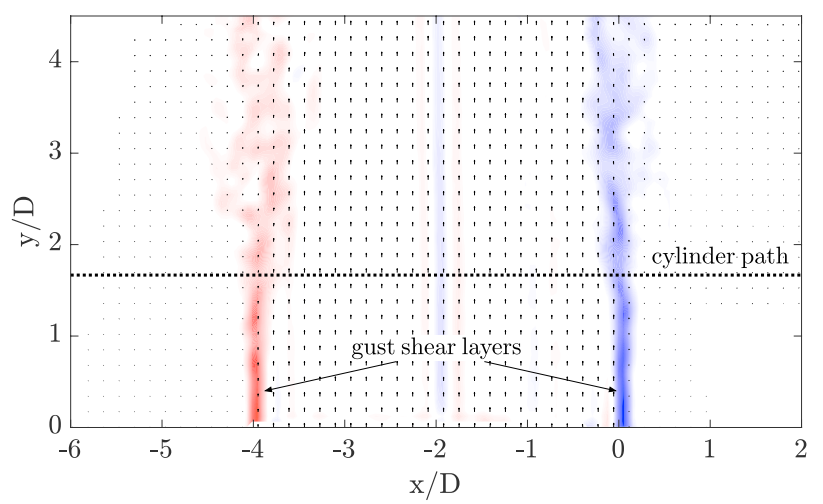

(a) Vorticity contours.

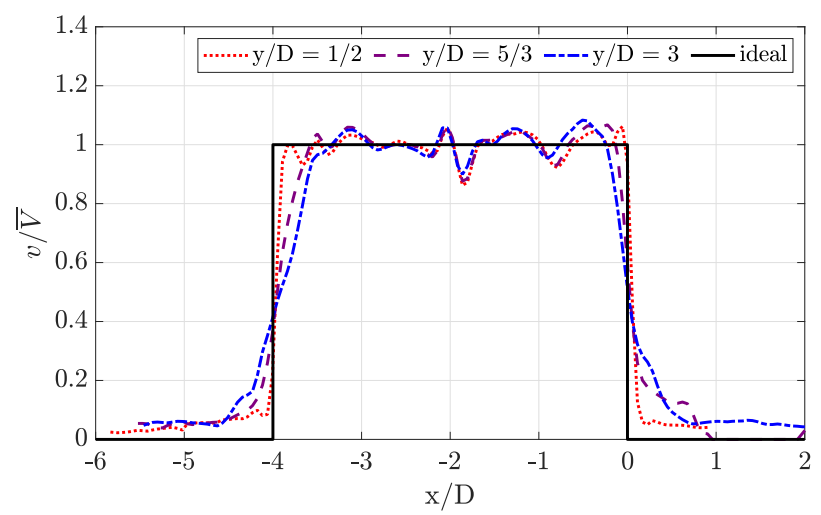

(b) Velocity profile of the gust at various vertical locations.

Fig. 9 Gust flow field. 


\section{Particle Image Velocimetry}

Planar particle image velocimetry (PIV) measurements are conducted at the cylinder midspan using an Nd:YLF $526 \mathrm{~nm}$ laser illuminating titanium dioxide particles dispersed in the water. Image acquisition is performed using two high speed Phantom M310 cameras. The resolution of each camera is $1280 \times 800$ pixels and the sampling rate is adjusted to $200 \mathrm{~Hz}$ and $800 \mathrm{~Hz}$ for the individual configurations. The test cases are repeated between 6 to 8 times and averaged thereafter. During the cross-correlation process, using the commercial LaVision Flowmaster 2D system, the interrogation window size is reduced from $64 \times 64$ pixels during its first pass to $16 \times 16$ pixels in its last, where an adaptive interrogation window is used with an overlap of $50 \%$. This results in an independent velocity vector spacing of $1.3 \mathrm{~mm}$ for the surging and rotating experiments in quiescent fluid and $3.4 \mathrm{~mm}$ during the cylinder gust encounter. The calculated vector fields for each camera are stitched together, yielding a final field of view $0.42 \mathrm{~m}$ wide and $0.13 \mathrm{~m}$ high for Cases 1 and 2 and $0.67 \mathrm{~m}$ by $0.37 \mathrm{~m}$ for Case 3 .

Two cameras and a horizontal dual light sheet, which is shone into the tank from either side, remove any shadow regions for the surge and rotation cases. When the gust rig is present, the light sheet from one side is blocked, creating a shadow region where no velocity data can be collected. The arrangements are visualised in figure 10. Both set-ups suffer from reflections of the laser sheet from the cylinder, leading to missing PIV data close to the surface. In a manner similar to [12], missing velocity data is recovered by interpolating between the measured flow field and the surface velocity of the cylinder. The latter is measured using a digital encoder on the EC motor and by an optical sensor tracking the carriage velocity. As a result, the magnitude of vorticity located directly around the cylinder is preserved. However, there is a small error in the surface normal distribution of the vorticity, as this cannot be accurately recovered. Since reflections only extend approximately $1-2 \mathrm{~mm}$ into the flow field, the associated error in the wall normal vorticity distribution is small and does not distort the results.

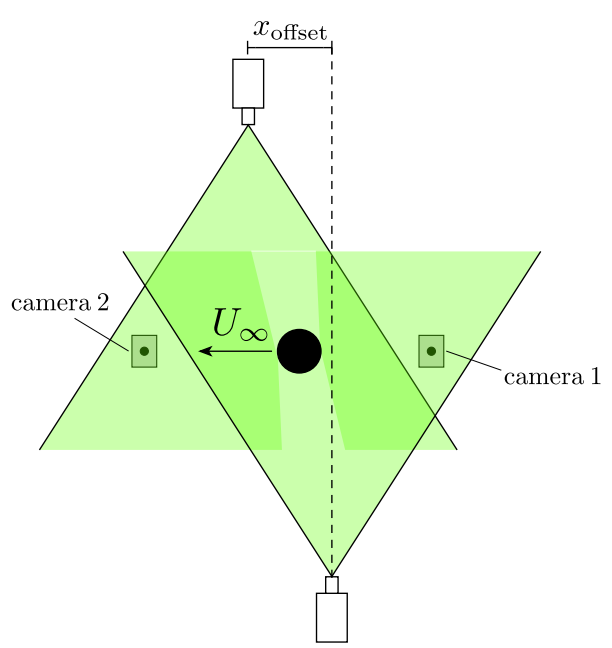

(a) Surging and rotating cylinder; no shadow region.

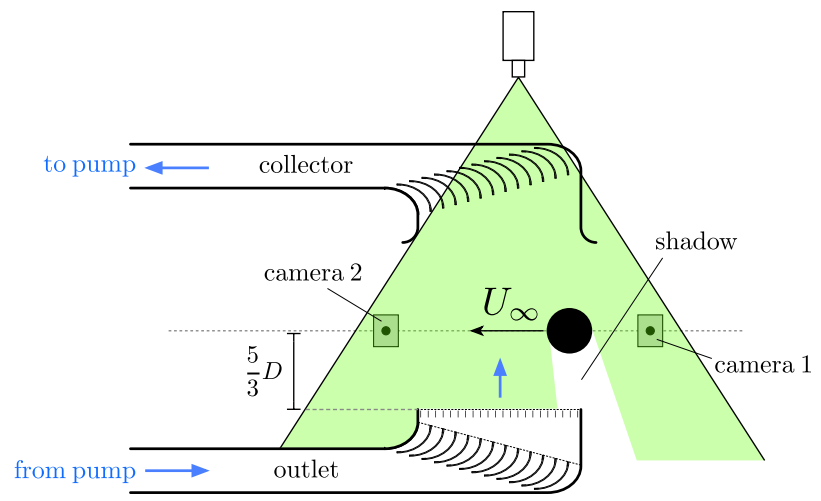

(b) Cylinder gust encounter; shadow region.

Fig. 10 Top view of the laser arrangement.

Peak locking effects can dominate the root mean square (RMS) velocity error estimation, due to particles being 'locked' to sensor pixels [22]. Ensuring that the illumination diameter of each particle is smeared over approximately two pixels reduces the associated RMS position error to 0.02 pixels, if sub-pixel interpolation is possible [23]. In the present study, the particle displacement is between 3 - 4 pixels. A conservative estimate therefore, suggests a velocity measurement error of $0.6 \%$. In addition, an associated error can be calculated by assessing the shift in the correlation peak when an interrogation window is mapped back to its original position according to the calculated displacement vector [24]. Small errors in this displacement vector will yield an offset that can be analysed. The uncertainty in the regions of interest, calculated by DaVis according to this method and reduced according to $1 / \sqrt{N}$ where $N$ is the number of samples [25], are $2 \%$ and $3 \%$ for the surging cylinder and the cylinder gust encounter, respectively. Therefore, the total error is below $3 \%$ and $4 \%$ for the two experimental set-ups. 


\section{B. Measuring Shed Circulation}

The method described in section $\Pi$ by which we predict the amount of circulation shed using the boundary layer flux will be referred to as the boundary layer method. The results from two measurement techniques, also computing the shed circulation, will be compared to the predictions from the boundary layer method. These two methods are used in conjunction to remove effects due to 3-dimensionality as well as from circulation leaving the observed flow field as much as possible.

The first method is a simple integration of all of the vorticity belonging to a vortex that is shed from the upper or lower surface of the cylinder. Any contribution from the boundary layer is excluded. 3-dimensional flow can develop in the wake, when the circulation has rolled up into a vortex and moved some distance away from the cylinder. This could affect the results by artificially reducing or increasing the measured circulation. Furthermore, once circulation leaves the flow field this method no longer yields correct results. Therefore, a second method is used. Here, the vorticity advected past a line is measured and integrated in time. This determines the shear layer 'feeding' the roll up. 3-dimensional affects are thought to be less of a problem here because they have not had sufficient time to develop. However, since the line is positioned some small distance away from the cylinder surface, there will be a small time delay between circulation shed from the surface and being advected past the line. Both methods are schematically illustrated in figure 11 .

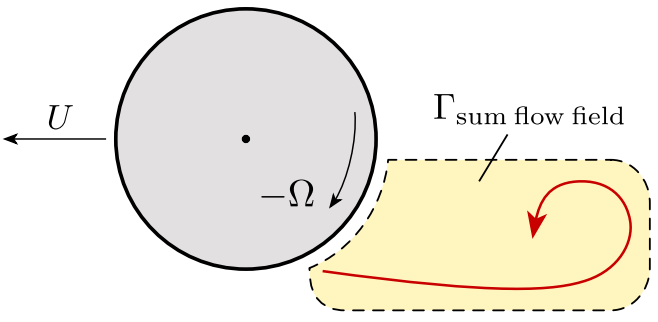

(a) Integration of $\Gamma$ in flow field.

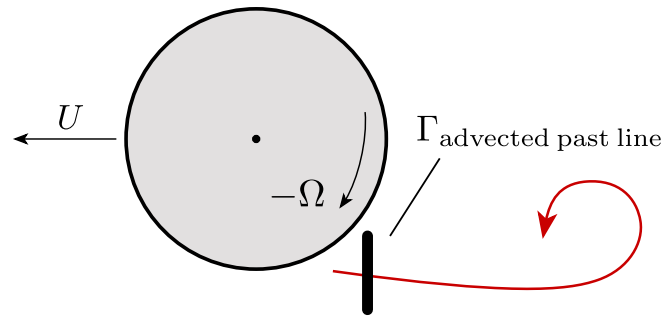

(b) Integration of $\Gamma$ advected past a line.

Fig. 11 Schematic illustration of how $\boldsymbol{\Gamma}_{\text {shed }}$ is computed in the flow field.

\section{Strength of Shed Circulation}

Prior to exploring how closely the boundary layer method matches direct measurements of the shed circulation, it is first tested whether it is possible to recover $\dot{\Gamma}_{b l}$ around the cylinder from PIV measurements. A comparison between the theoretical vorticity flux obtained from potential flow, when the cylinder is surging as well as surging and rotating, confirms that this is possible, as demonstrated in figures 12 and 13 . The theoretical results match the experimental measurements as long as no separation has occurred. Thereafter, as to be expected, the potential flow and experimental vorticity flux differ, as $\dot{\Gamma}_{b l}$ is now impacted by $\gamma_{c}$, which is disregarded in the theoretical prediction.

Satisfied that the resolution of the PIV vorticity vectors is sufficient to measure $\dot{\Gamma}_{b l}$ and that the problems associated with laser sheet reflections do not adversely affect the results, the amount of shed circulation can be evaluated. The comparison between using the boundary layer method, where $\dot{\Gamma}_{\text {shed }}$ is integrated in time to yield the amount of shed circulation, and the two standard methods described in section III.B for the purely translating cylinder as well as for the translating and rotating case are shown in figure 14.

When vortex shedding begins, the amount of circulation shed into the flow appears to increase linearly for the surging cylinder. This is observed to occur simultaneously from both sides of the cylinder. When the cylinder is rotated, vortex shedding no longer begins at the same time from both sides. Circulation is first shed from the lower surface. Initially the amount of circulation shed appears to increase linearly but deviates from this at later stages. Negative circulation is shed from the upper surface when $s / D>2.7$. Once more an approximately linear increase in magnitude is observed.

When comparing the individual methods measuring how much circulation is shed, it is observed that:

- There is a good agreement between the different methods when $s / D<4$ in both cases. 


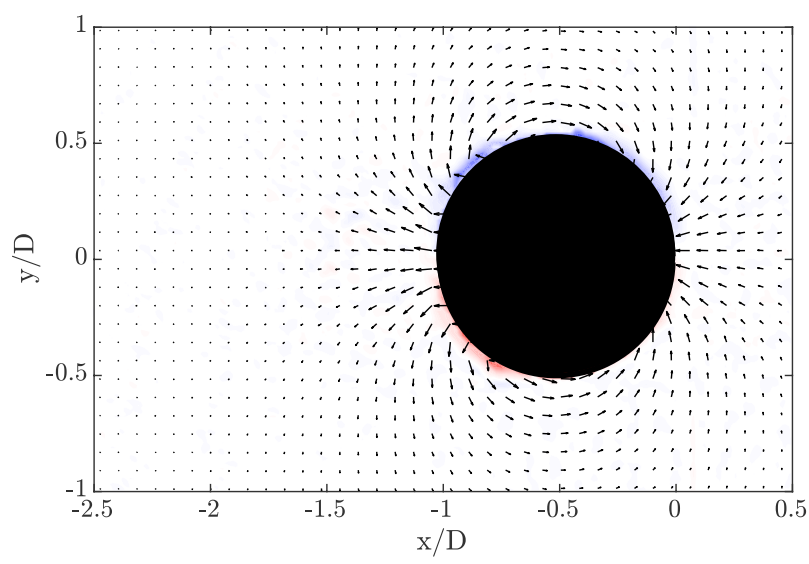

(a) $s / D=0.5$

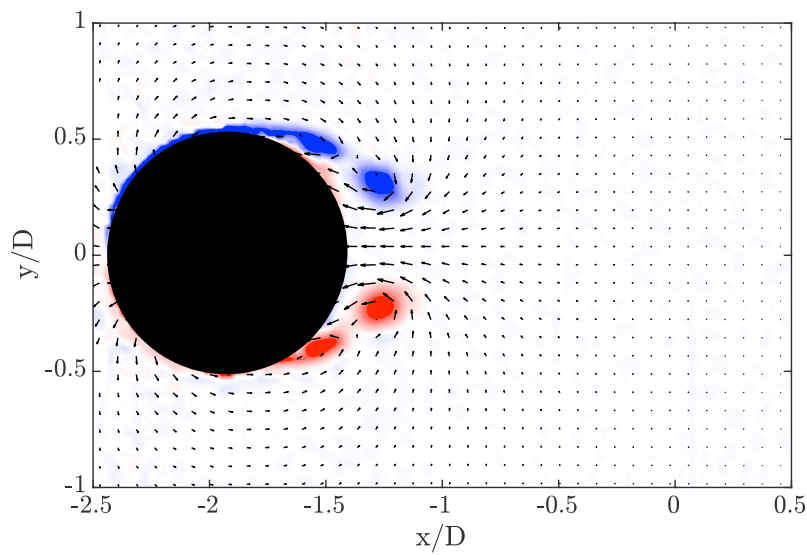

(c) $s / D=1.9$

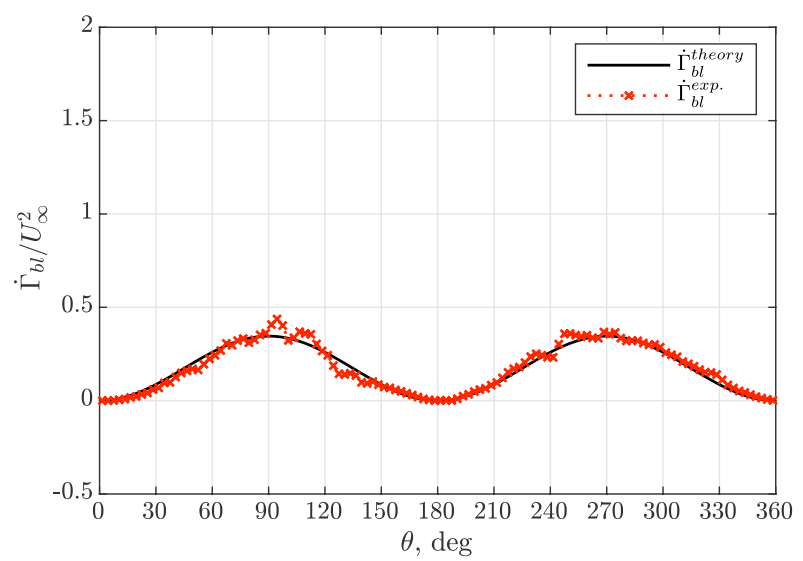

(b) $\dot{\Gamma}_{b l}$ matches the potential flow prediction.

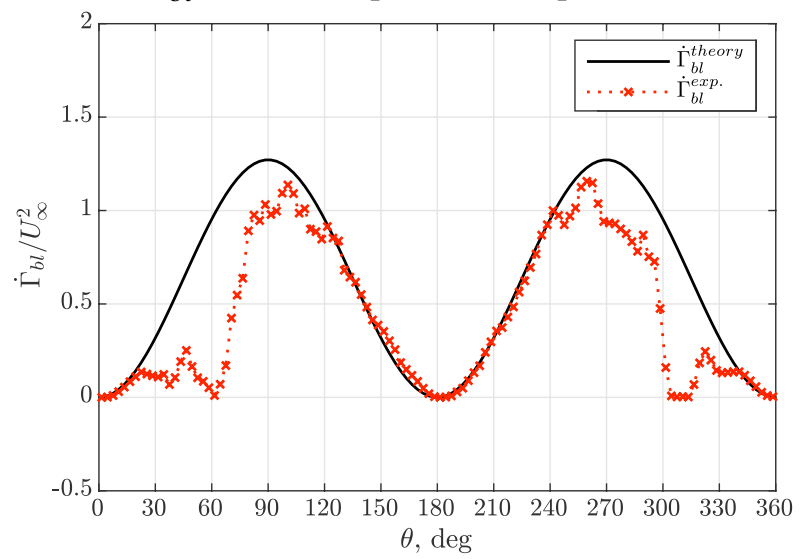

(d) $\dot{\Gamma}_{b l}$ differs to potential flow prediction due to separation.

Fig. 12 Normalised vorticity contours and vorticity flux. Surge only, Case 1.

- After $s / D=4$ there is a deviation between the boundary layer method prediction and the shed circulation obtained from integrating $\Gamma$ in the flow field. For the surging cylinder this suggests that 3 -dimensionality in the wake is affecting the 2-dimensional planar PIV measurements, as no circulation has left the field of view. For the rotating and surging cylinder, positive circulation shed from the lower half of the cylinder begins to leave the observed flow field just prior to $s / D=4$; figure $17 \mathrm{~b}$. Necessarily, this causes an incorrect measurement of $\Gamma$. When comparing the boundary layer method to $\Gamma_{\text {shed }}$ obtained by integrating $\Gamma$ advected past a line, this discrepancy does not arise. Here, 3-dimensional effects or circulation leaving the flow field do not affect the results.

- The circulation obtained by integrating the circulation advected past a line deviates from the two other methods during the initial phase of vortex shedding for the rotating case. This is because it takes a finite amount of time for the circulation to travel to and advect past the line. Furthermore, difficulty in placing the line at the correct location yields a further small discrepancy for the positive circulation shed in the rotating case.

The two cases involving a surging and rotating cylinder in quiescent fluid could be classified as relatively basic and standard flow fields, that have already been subjected to much investigation. Therefore, the more complex scenario of a surging clockwise rotating cylinder entering a sharp-edged gust is explored. The negative circulation shed from the upper surface, evaluated using the boundary layer method, is compared to an integration of the respective circulation observed in the flow field. Figure 15 provides a snapshot of the normalised vorticity contours of the cylinder gust encounter together with the comparison of the calculated shed circulation. Once more an excellent match between the different methods is obtained. Analysing the circulation shed from the bottom half the cylinder, was unfortunately not possible, due to the shadow region caused by the cylinder. 


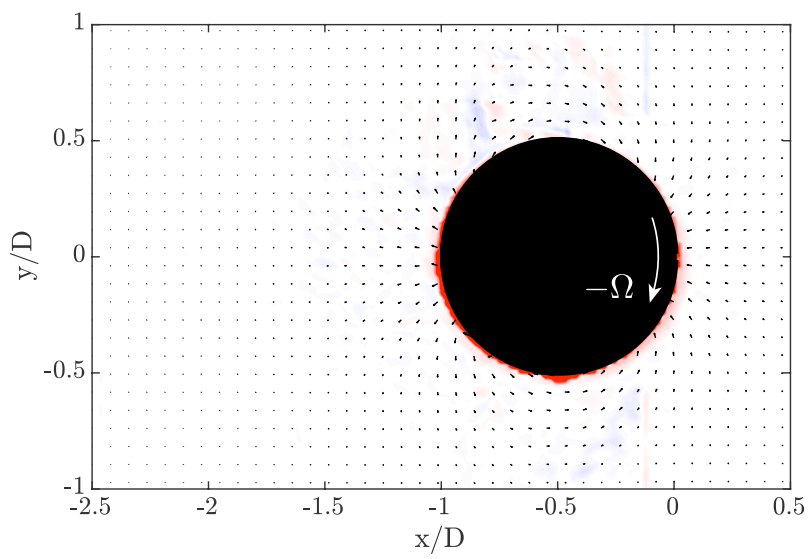

(a) $s / D=0.5$

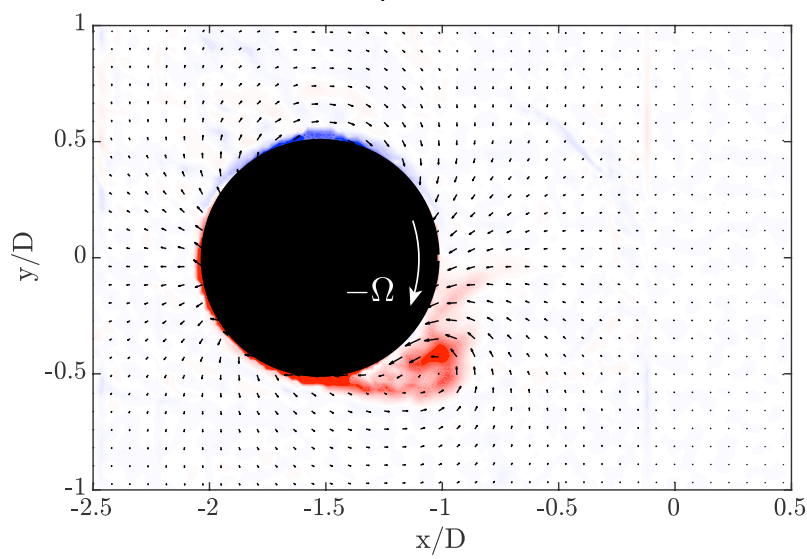

(c) $s / D=1.5$

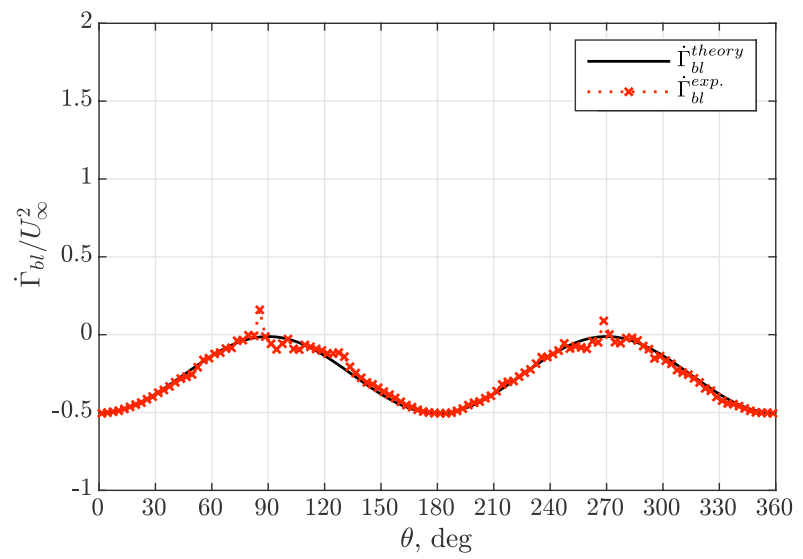

(b) $\dot{\boldsymbol{\Gamma}}_{b l}$ matches the potential flow prediction.

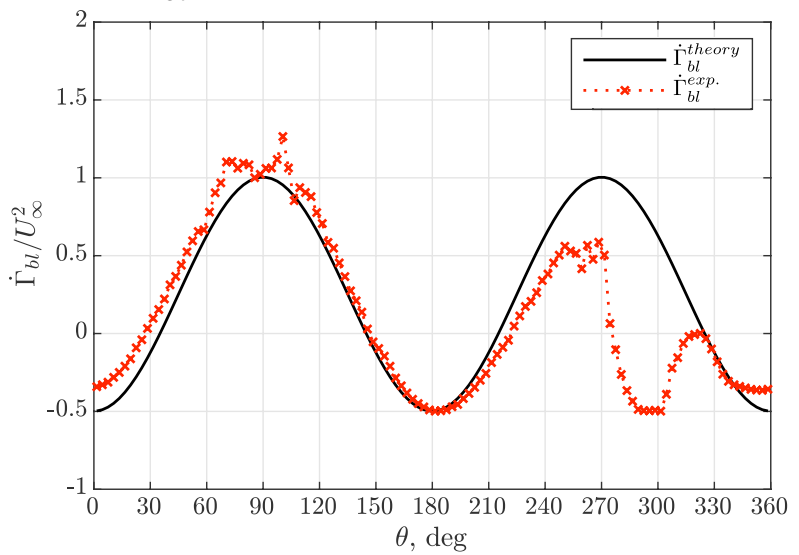

(d) $\dot{\Gamma}_{b l}$ differs to potential flow prediction due to separation.

Fig. 13 Normalised vorticity contours and vorticity flux. Surge and rotation, $\alpha=1$. Case 2.

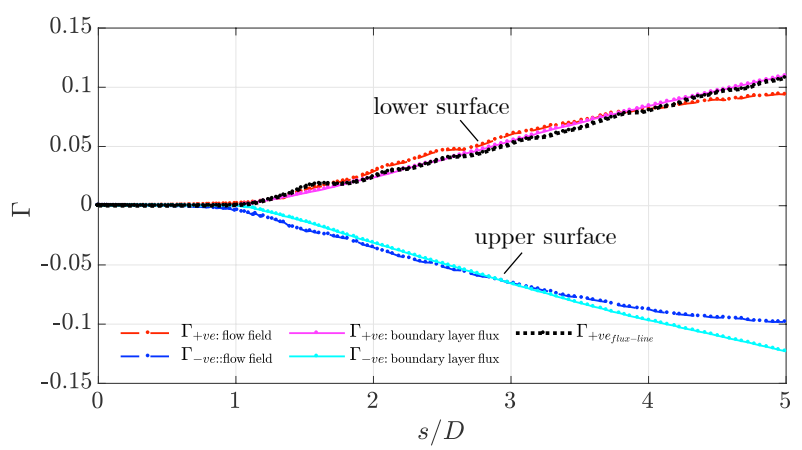

(a) Translating, $S_{a c c}=3$, Case 1 .

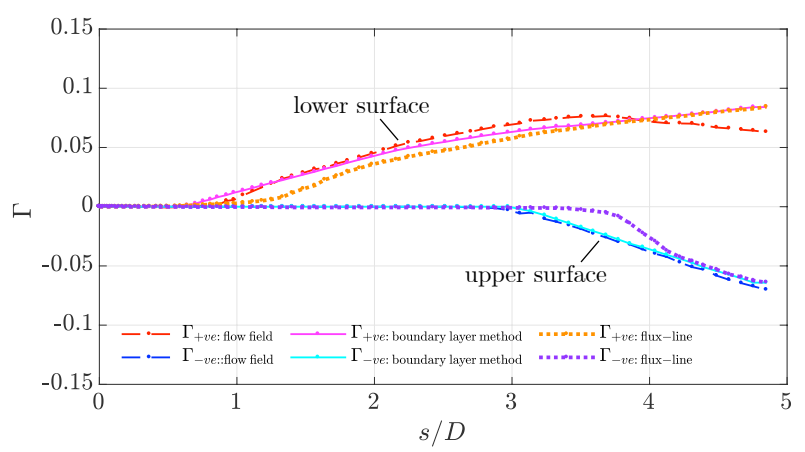

(b) Translating and rotating, $\alpha=1, S_{a c c}=2$, Case 2 .

Fig. 14 Comparison of computing $\Gamma_{\text {shed }}$ by using the boundary layer method and by directly interrogating the vorticity field.

The results therefore indicate that it is possible to determine $\Gamma_{\text {shed }}$ purely from evaluating the boundary layer vortex sheet strength at the point of separation. 


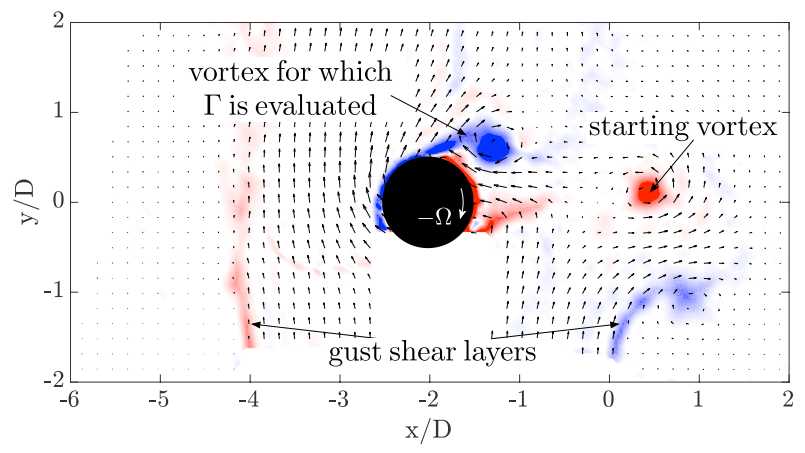

(a) Vorticity contours.

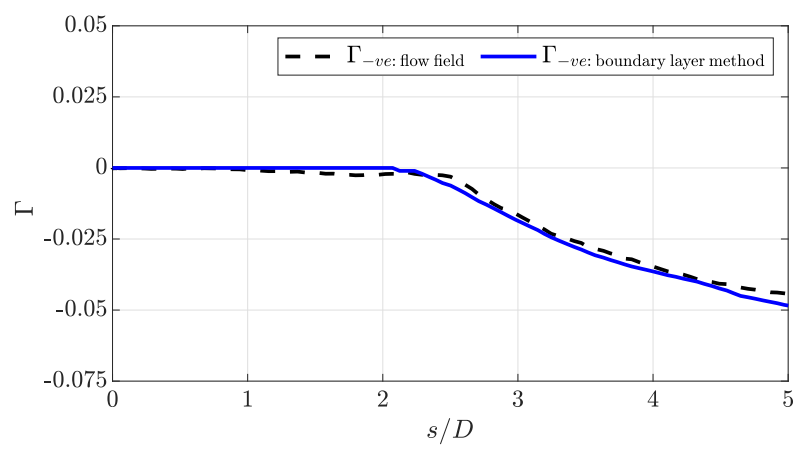

(b) Shed circulation.

Fig. 15 Comparison of computing $\Gamma_{\text {shed }}$ by using the boundary layer method to directly interrogating the vorticity field, during a cylinder gust interaction. Case 3

\section{Shedding Rate of Circulation}

Figure 16 shows the variation of $\dot{\Gamma}_{\text {shed }}$ for the surging cylinder. Shedding begins simultaneously from both sides of the cylinder and increases until the end of the acceleration region at $s / D=3$. After this, the shedding rate remains approximately the same for the remainder of the captured motion, albeit with some small oscillations. Non-dimensionalizing $\dot{\Gamma}_{\text {shed }}$ with the instantaneous velocity, $U$, rather than $U_{\infty}$, causes the shedding rate to remain approximately constant for $s / D>1.2$. $\dot{\Gamma}_{\text {shed }}$ from either side of the cylinder is almost identical, indicated by each pair of lines lying nearly on top of one another. This appears reasonable, as the observed flow field is symmetrical.

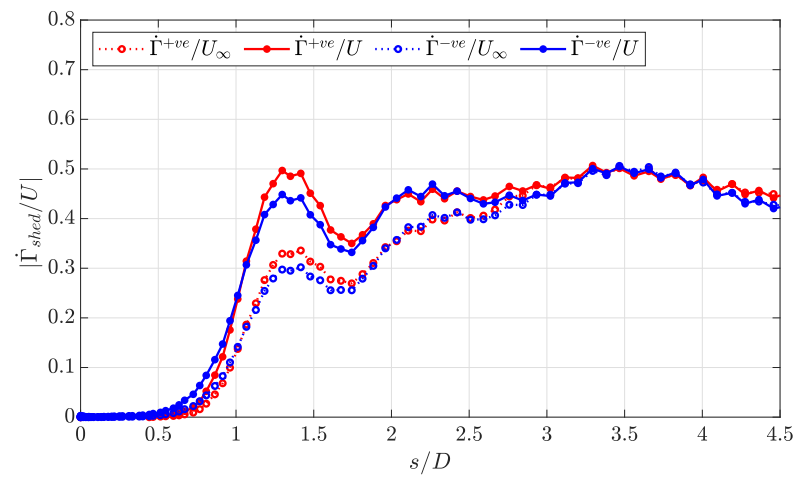

(a) Translating, $S_{\text {acc }}=3$, Case 1 .

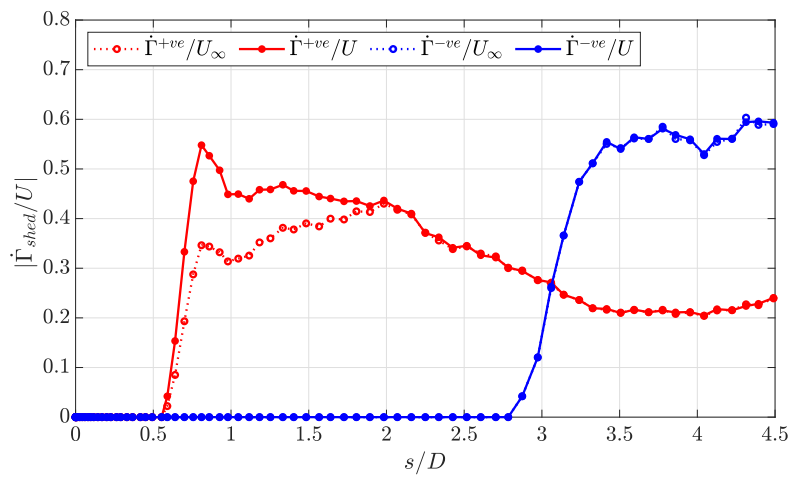

(b) Translating and rotating, $\alpha=1, S_{a c c}=2$, Case 2.

Fig. 16 Non-dimensional rate at which circulation is shed. Scaling with instantaneous velocity causes $\dot{\Gamma}_{\text {shed }}$ to remain constant for the surging case. The rotating and translating cylinder shows both a constant and varying shedding rate. Absolute values are plotted.

The shedding rate for the surging and rotating cylinder is depicted in figure $16 \mathrm{~b}$. Positive circulation begins to be shed from the lower surface before $s / D=1$. The flow field, when separation has just started is shown in figure 17a Broadly, its development can be grouped up into three phases:

1) After an initial sharp increase, $\dot{\Gamma}_{\text {shed }}$ increases more gradually until the end of acceleration at $s / D=2$. Nondimensionalizing with $U$, causes $\dot{\Gamma}_{\text {shed }}$ to remain approximately constant in this region, albeit with a small downwards slope.

2) For greater translation distances, between $2<s / D<3.5$, there is a gradient change and the shedding rate reduces. This coincides with the starting vortex advecting away from the cylinder and the shed circulation forming a separating shear layer.

3) After $s / D=3$, circulation shedding assumes a constant rate once again. An example of the flow field during this stage is shown in figure $17 \mathrm{~b}$ 
Negative circulation is shed from the upper surface when $s / D>2.7$; here $U$ is constant and equal to $U_{\infty}$. Throughout the observed field of view, the shedding rate remains constant.

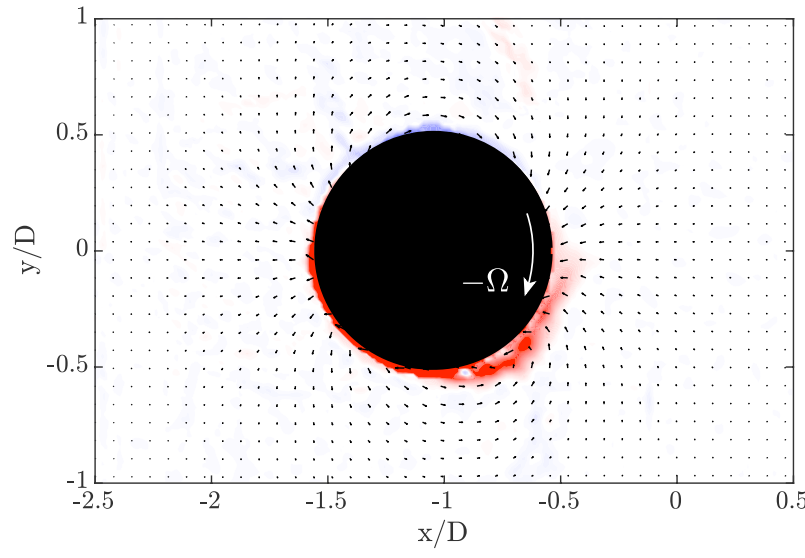

(a) $s / D=1.04$. Separation has just begun.

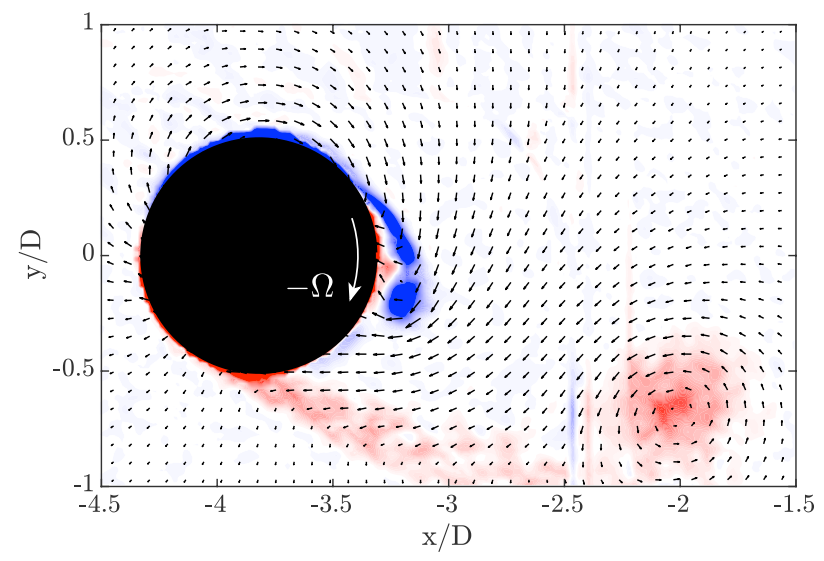

(b) $s / D=3.82$. Shed circulation has left the flow field.

Fig. 17 Normalised vorticity contours. Surge and rotation, $\alpha=1$. Case 2.

Two key observations can be made from figure 16 The first one regards the shedding rate during the acceleration region. From equation 12, $\dot{\Gamma}_{\text {shed }}$ is only a function $\gamma_{n c}$ and $\gamma_{c} \cdot \gamma_{n c}$ itself scales directly with instantaneous velocity. As the cylinder accelerates, $\gamma_{n c}$ grows and thus directly affects the rate at which circulation is shed. Therefore, scaling the shedding rate by $U$ causes it to remain approximately constant during the initial translation period. This agrees with observations by Manar et al. [19] who found that the amount of shed circulation for different kinematic cases of an accelerating flat plate collapses, if the shed circulation is normalised by instantaneous velocity [19].

The second observation from figure 16 is that the rate at which circulation is shed is not entirely different between the purely surging and the simultaneously rotating and surging case. The shedding rate is also not strongly dependent on whether the cylinder wall is moving with or against the local flow. This is especially clear just after circulation shedding has started. Here, $\dot{\Gamma}_{\text {shed }}$ is least affected by $\gamma_{c}$. It demonstrates that the rotation rate only shifts the separation point and indirectly affects $\gamma_{c}$, but that the shedding rate does not explicitly scale with rotation.

\section{E. Variation in Shedding Rate}

It is observed that the rate at which circulation is shed into the flow field is constant during certain times and varies at others. To explore why this occurs, we can make use of the fact that $\dot{\Gamma}_{\text {shed }}$ is a function of $\gamma_{b}$. Since $\gamma_{b}$ is composed of $\gamma_{n c}, \gamma_{r}$ and $\gamma_{c}$ the variation of the individual vortex sheets can be analysed to inform what causes the observed change in $\dot{\Gamma}_{\text {shed }}$. During the kinematic steady state region both $\gamma_{n c}$ and $\gamma_{r}$ remain constant. They can therefore, not be the reason as to why $\gamma_{b}^{\text {sep }}$. varies and consequently, this only leaves $\gamma_{c}$ as a possible culprit.

$\gamma_{c}$ is governed by the location and strength of shed circulation. If circulation resides close to the cylinder surface, then the effect of $\gamma_{c}$ is confined to a local region along the cylinder surface. This is because the induced velocities from the shed vortex and its mirror counterpart will be in the same direction. On the opposite side of the cylinder, however, the individual velocities are of the opposite sign. Furthermore, the location of the shed and mirror vortex is very similar if the shed vortex is close to the cylinder surface and thus the opposing velocities cancel. This is schematically shown in figure 18 . In contrast, when the shed circulation has advected infinitely far away from the cylinder, the mirror image is located at the cylinder centre and generates a vortex sheet of equal strength all along the cylinder.

During a developing unsteady separation, circulation is continuously shed from the surface. Simultaneously, for greater translation distances, some circulation has advected far away. In such a case, the vortex sheet is affected locally by circulation that has just been shed and globally by circulation at infinity. This is demonstrated in figure 19 , where the blue dashed line indicates the strength of the vortex sheet around the cylinder.

Consider a cylinder that has just begun to surge from the right to the left as well as to rotate clockwise after having 


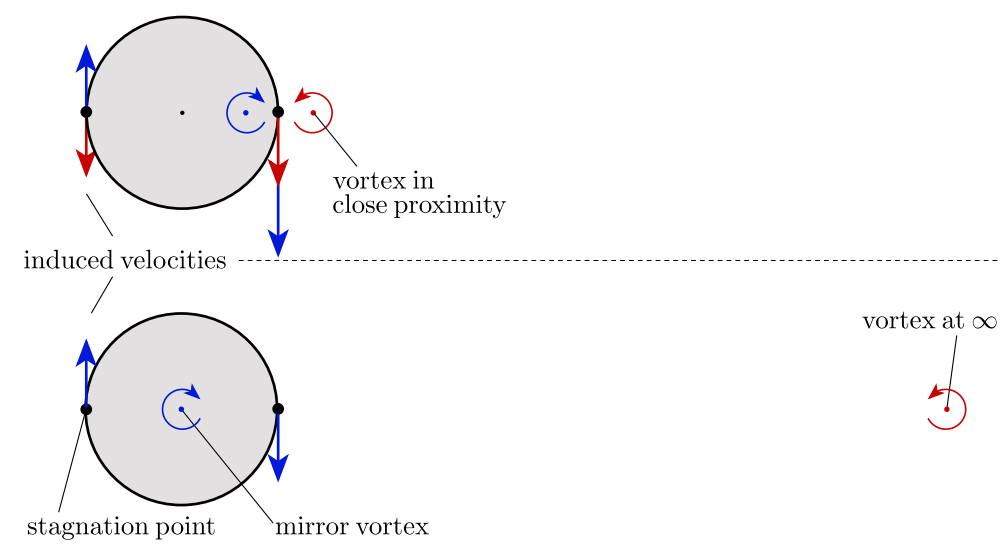

Fig. 18 Induced velocities by a vortex in close proximity to the surface and one located at infinity.

initially been stationary. Before any separation occurs, $\gamma_{b}$ is made up of $\gamma_{n c}$ and $\gamma_{r}$ only. As the cylinder moves, circulation begins to shed into the flow field and $\gamma_{c}$ is no longer zero. This is step 1 in figure 20 The corresponding vortex sheet shows a drop after the separation point and since the shed circulation is located close to the cylinder $\gamma_{c}$ is non-zero locally. As demonstrated previously, the strength of the shed separation is proportional to $\gamma_{b}^{\text {sep. }}-\gamma_{r}^{\text {sep. }}$ or equivalently $\gamma_{n c}^{\text {sep. }}+\gamma_{c}^{\text {sep. }}$. Eventually, the starting vortex advects far away from the cylinder. This causes $\gamma_{c}$ to be of equal strength all along the cylinder, as the corresponding mirror image of the shed starting vortex moves towards the cylinder centre, step 2 in figure 20. The global reduction of $\gamma_{c}$ shifts $\gamma_{b}$ downwards and consequently reduces $\gamma_{b}^{\text {sep. }}-\gamma_{r}^{\text {sep. }}$ and thus the amount of circulation shed, as observed in figure $16 \mathrm{~b}$

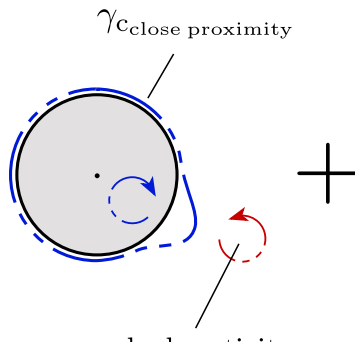

shed vorticity in close proximity

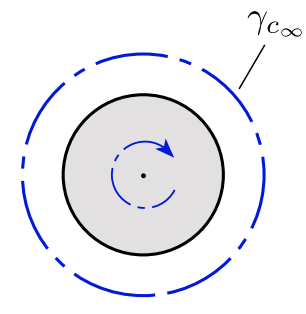

starting vortex at $\infty$

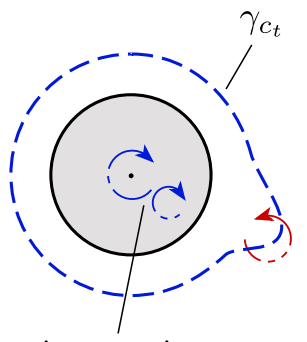

mirror vortices

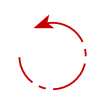

Fig. 19 Illustrating the effect of shed circulation located in close proximity to the cylinder and that of circulation advected to infinity on $\gamma_{c}$. The strength of the vortex sheet is indicated by the distance between $\gamma$ and the cylinder surface.

An element of shed circulation has a flow field associated with it, that is of a circular nature. Ignoring any viscous effects, it is equivalent to that of a point vortex. The entire flow field is a superposition of such velocity distributions due to shed circulation as well as due to uniform horizontal freestream. If the elements of shed circulation are strong enough, relative to the freestream, then the flow field is dominated by the induced rotational velocity. Therefore, if during unsteady separation, the strength of the shed circulation is sufficient, the rotational flow field dominates locally and the shed circulation rolls up into a coherent vortex. However, if the strength of the shed circulation reduces, then the relative strength between the rotational flow field associated with the shed circulation, compared to the freestream velocity, is too weak a create a coherent rolled up vortex and the circulation sheds as a shear layer.

The downwards shift of $\gamma_{b}$ has a further consequence. For $\alpha<2$, negative circulation begins to be created on the upper cylinder surface. If $\gamma_{b}$ is shifted downwards sufficiently, a local critical negative value of $\gamma_{b}$ is reached, as hypothesised in [12], initiating flow separation from the upper surface; step 3 in figure 20 This secondary separation vortex advects downstream. As it moves to infinity, the mirror image moves closer to the cylinder centre and begins to negate the effect of the mirror image of the starting vortex. With the two mirror images cancelling, $\gamma_{c}$ returns to zero and in turn $\gamma_{b}$ shifts upwards, step 4. This results in a less negative value of $\gamma_{b}^{\text {sep. }}-\gamma_{r}^{\text {sep. }}$ on the upper surface, reducing the 

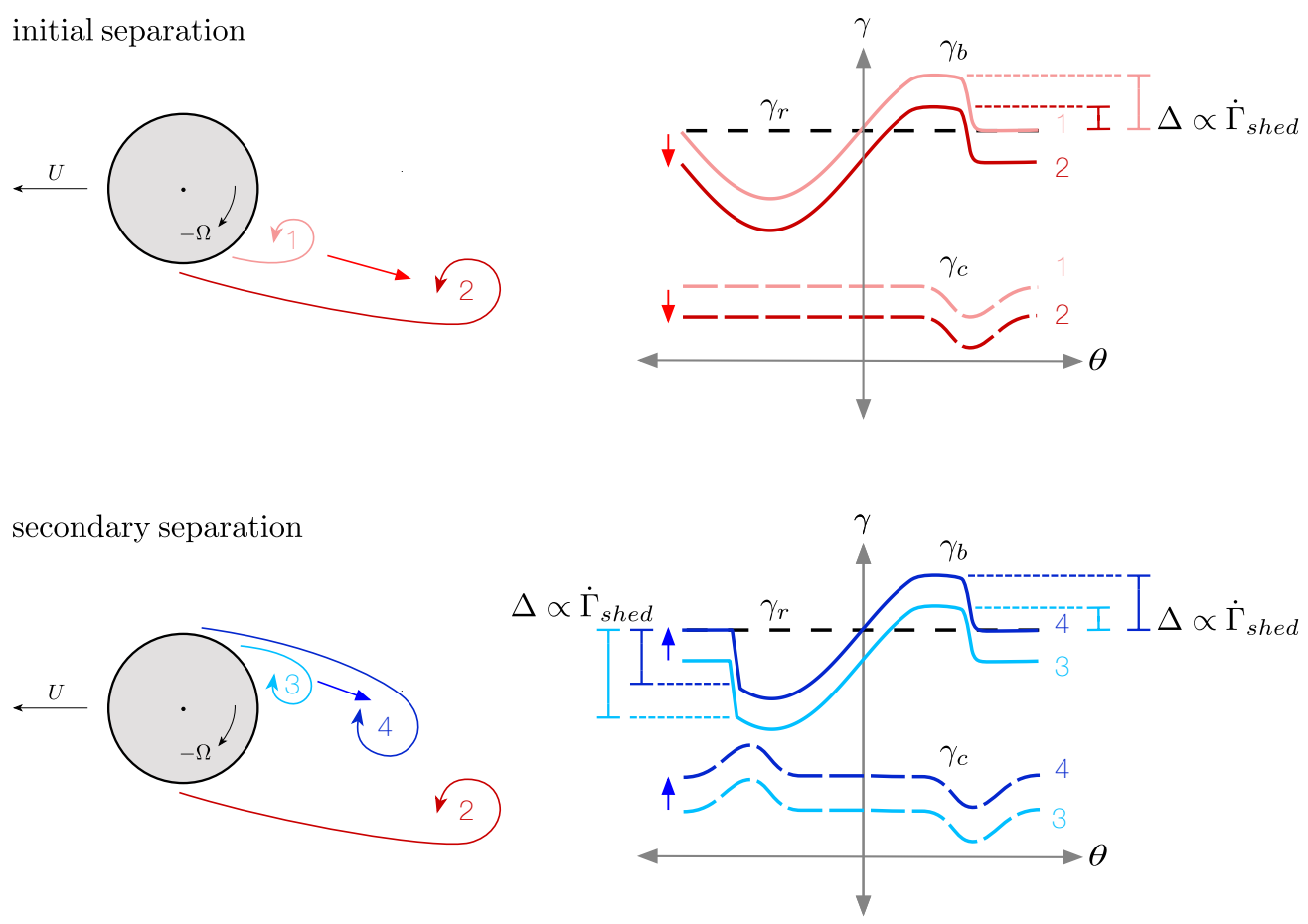

Fig. 20 Flow field and $\gamma_{b}$ and $\gamma_{c}$ development for a surging and clockwise rotating cylinder. Each number corresponds to one instance in time, with the colours matching accordingly.

amount of circulation shed, whilst increasing $\gamma_{b}^{\text {sep. }}-\gamma_{r}^{\text {sep. }}$ on the lower side. In turn, the circulation shed from the upper surface no longer forms a coherent vortex but sheds in form of a shear layer or seizes altogether. On the lower side, the increased strength in shed circulation induces sufficient rotational velocity to cause the shed circulation to roll up in a new coherent vortex.

To prove the outlined hypothesis, $\dot{\Gamma}_{\text {shed }}$ is computed according to equation 12 for the surging and rotating cylinder case. Initially, no correction for circulation advecting to infinity is applied and the raw measurement data is used. The result of this was presented in figure $16 \mathrm{~b}$ Between $2.5<s / D<3.5$ a noticeable change in $\dot{\Gamma}_{\text {shed }}$ is observed. Evaluating the strength of $\gamma_{c}$ at the stagnation point, $\gamma_{c}^{\text {stag. }}$, is used to give an estimate of the effect of circulation that has advected to infinity and its 'global' effect on the vortex sheet. It is assumed that circulation in close proximity to the cylinder surface will induce a negligible $\gamma_{c}$ at the stagnation point, due to the cancellation of induced velocities by itself and its mirror image, figure 18 .

Re-calculating $\dot{\Gamma}_{\text {shed }}$ corrected by $\gamma_{c}^{\text {stag. }}$

$$
\dot{\Gamma}_{\text {cor. }}=\frac{\left(\gamma_{b}^{\text {sep. }}-\gamma_{c}^{\text {stag. }}-\gamma_{r}^{\text {sep. }}\right)^{2}}{2}
$$

and normalising by instantaneous velocity causes $\dot{\Gamma}_{c o r}$. on both sides of the cylinder to remain approximately constant, figure 21. This is observed throughout the entire measured surging motion and hence for a vastly and rapidly changing flow field. Of course the result of $\dot{\Gamma}_{c o r}$, after the correction is applied, no longer corresponds to the actual $\dot{\Gamma}_{\text {shed }}$ that can be used to estimate the amount of shed circulation; but that is not the intended result. Rather, a constant strength of $\dot{\Gamma}_{c o r}$. strongly suggests that the reason behind the observed variation in shedding rate is caused by circulation advecting to infinity.

For the purely surging cylinder, two attached vortices form on either side of the cylinder during the captured translation distance. The symmetry of the flow field, two vortices of equal magnitude but opposite strength, causes $\gamma_{c}^{\text {stag. }}$ to remain zero and thus $\dot{\Gamma}_{\text {cor }}$ has not been included for this case, as it would look identical to figure $16 \mathrm{a}$ 


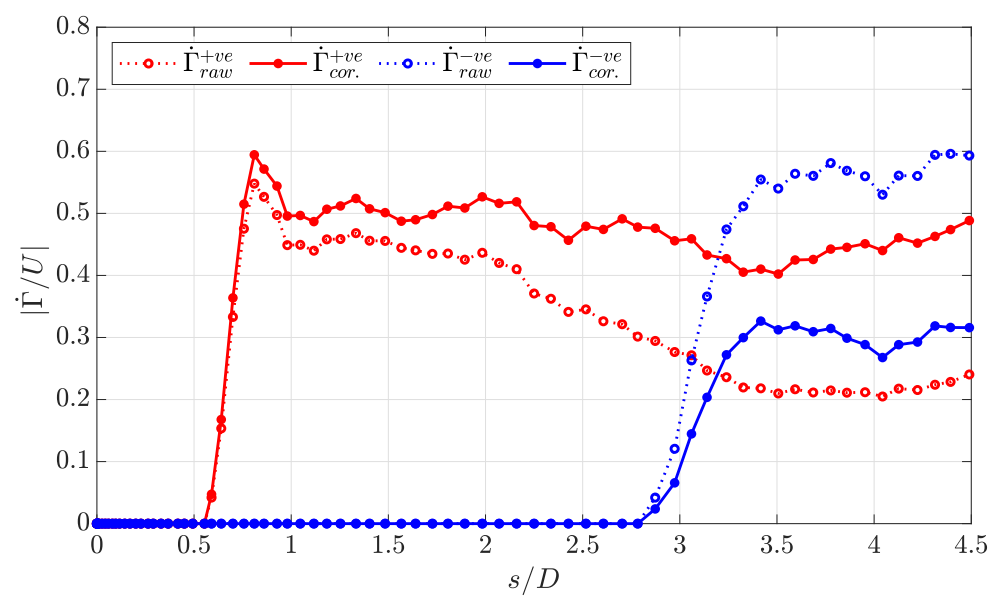

Fig. 21 Accounting for circulation advecting to infinity. Surging and rotating cylinder, Case 2.

\section{F. Maximum Theoretical $C_{l}$}

As a final consideration to reconcile the proposed theory with existing results, the relation

$$
\dot{\Gamma}_{\text {shed }}=f\left(\gamma_{b}^{\text {sep. }}, \gamma_{r}^{\text {sep }}\right)=f\left(\gamma_{n c}^{\text {sep. }}, \gamma_{c}^{\text {sep. }}\right),
$$

can be applied to Prandtl's upper limit on the theoretical lift coefficient, $C_{l}^{\max }$, for a surging and rotating cylinder [26]. Prandtl and Goldstein [18] argued that $C_{l}^{\max }$ is reached when the stagnation points collapse and move from the surface into the flow. Since the streamlines form a closed loop around the cylinder, the shedding of any further circulation is prevented. It must be noted that this upper limit is only of theoretical nature, as it disregards any potential instabilities. Hence, it effectively considers a potential flow. For example Tokumaru \& Dimotakis [27] exceeded this theoretical value by more than $20 \%$.

However, the theoretical limit of Prandtl can be recovered by linking the shedding rate to the difference between $\gamma_{b}^{\text {sep }}$. and $\gamma_{r}^{\text {sep. }}$ as per equation 12 , when similarly considering such an idealised flow. When the difference between $\gamma_{b}^{\text {sep. }}$ and $\gamma_{r}^{\text {sep. }}$ is zero, no more circulation is shed. Since $\gamma_{b}-\gamma_{r}$ is equivalent to $\gamma_{n c}+\gamma_{c}$, shedding should seize when $\gamma_{c}^{\text {sep. }}=-\gamma_{n c}^{\text {sep. }}$. The latest this could occur is when $\gamma_{c}^{\text {sep. }}$ is equal to the maximum of $\gamma_{n c}^{\text {sep. }}$. For a surging cylinder this would occur at

$$
\gamma_{c}^{\text {sep. }}=-2 U \text {. }
$$

Imagine a cylinder surging from right to left whilst simultaneously rotating clockwise. From a stationary start a starting vortex is shed. This advects to infinity relative to the cylinder. Once the shed circulation reaches a strength of $4 \pi U a$, where all of it is located at infinity, $\gamma_{c}=-2 U$ everywhere. Hence $\gamma_{c}^{\text {sep. }}=-\gamma_{n c}^{\text {sep. }}$ and shedding seizes. A schematic illustration of the vortex sheet strengths reflecting this behaviour is shown in figure 22 The result is equivalent to that obtained by Prandtl but derived purely from a vortex sheet consideration.

\section{Conclusion}

A surging and rotating cylinder in quiescent fluid as well as a sharp-edged gust encounter is used to investigate the resulting unsteady shedding behaviour of circulation. Evaluating the boundary layer at the separation point has given insight into the parameters affecting the shedding strength of circulation and its respective variation. A possible alternative explanation for the development of an alternating vortex shedding pattern has also been proposed. The key results presented in this investigation are:

- The vorticity flux around the surface of a body can be measured experimentally. It matches that predicted by potential flow, when no flow separation has occurred, and only depends on the boundary layer vortex sheet components. 


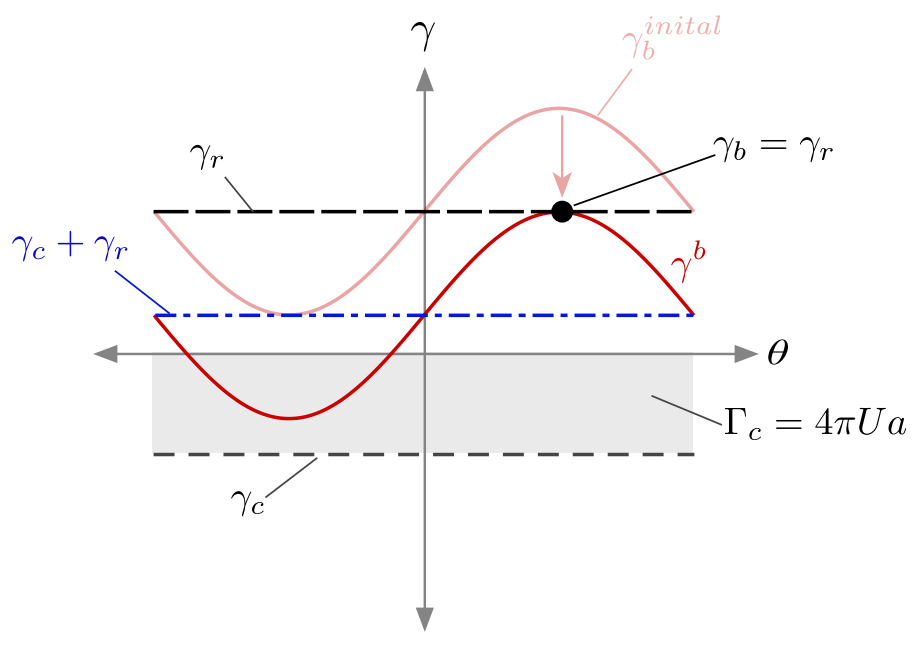

Fig. 22 A vortex at infinity with $\Gamma=4 \pi U a$ causes $\gamma_{b}$ to equal $\gamma_{r}$ hence $\dot{\Gamma}_{\text {shed }}=0$.

- The amount of circulation shed at the separation point was shown to be equivalent to the vorticity flux in the boundary layer for each of the test cases. This suggests that it is possible to predict the amount of circulation shed during unsteady separation from a knowledge of the surface vortex sheet strength. At the first onset of separation, the vortex sheet strength can be determined from simple potential flow theory. Thus the shedding rate, once separation occurs, can be predicted from the knowledge of the location of separation. For a spinning cylinder, the rotation rate has no direct effect on the rate at which circulation is shed, however, the spin rate does influence where and when separation occurs.

- Circulation advecting to infinity causes a uniform shift of the boundary layer vortex sheet strength. This can increase or decrease the rate at which circulation is shed. The induced rotational velocity field, due to any shed circulation, varies accordingly. If the induced rotational velocity dominates, then a local rotating flow is created causing the shed circulation to form as a coherent vortex. If the circulation shed is too weak, the local flow velocity will rather be dominated by the uniform freestream and the circulation will shed in form of a shear layer. Therefore, understanding why the strength of shed circulation varies explains why circulation sometimes rolls up into a vortex and at other times sheds in form of shear layer. The effect of circulation at infinity may also explain why vortices are shed in an alternating pattern from either side of the cylinder, without needing to invoke a qualitative "effective change of the body shape' argument.

- Prandtl's result, although non-physical, suggesting that the theoretical maximum lift coefficient, for a surging and rotating cylinder, is $4 \pi$ can be recovered using only the dependency of the shedding rate on the difference between $\gamma_{b}$ and $\gamma_{r}$.

\section{Acknowledgments}

The author would like to acknowledge the Engineering and Physical Science Research Council (EPSRC) for providing financial support. 


\section{References}

[1] Stevens, P. R. R. J., “Unsteady Low Reynolds Number Aerodynamic Forces,”, No. April, 2015.

[2] Stevens, P. R. R. J., and Babinsky, H., "Experiments to Investigate Lift Production Mechanisms on Pitching Flat Plates," Experiments in Fluids, Vol. 58, No. 1, 2017, pp. 1-17.

[3] Babinsky, H., and Stevens, P. R. R. J., "Low Order Modelling of Lift Forces for Unsteady Pitching and Surging Wings ." AIAA, 2016, pp. 1-12.

[4] Eldredge, J. D., and Jones, A. R., "Leading-Edge Vortices: Mechanics and Modeling," Annual Review of Fluid Mechanics, Vol. 51, No. 1, 2019, pp. 75-104.

[5] Watkins, S., Milbank, J., Loxton, B. J., and Melbourne, W. H., "Atmospheric Winds and Their Implications for Microair Vehicles," AIAA Journal, Vol. 44, No. 11, 2006, pp. 2591-2600.

[6] Watkins, S., Thompson, M., Loxton, B., and Abdulrahim, M., "On Low Altitude Flight Through The Atmospheric Boundary Layer," International Journal of Micro Air Vehicles, Vol. 2, No. 2, 2010, pp. 55-68.

[7] White, C., Lim, E. W., Watkins, S., Mohamed, A., and Thompson, M., “A Feasibility Study of Micro Air Vehicles Soaring Tall Buildings," Journal of Wind Engineering and Industrial Aerodynamics, Vol. 103, 2012, pp. 41-49.

[8] Saffman, P. G., and Schatzman, J. C., "An Inviscid Model for the Vortex-Street Wake,” Journal of Fluid Mechanics, Vol. 122, 1982, pp. 467-486.

[9] Eldredge, J. D., "A Reconciliation of Viscous and Inviscid Approaches to Computing Locomotion of Deforming Bodies," Experimental Mechanics, Vol. 50, No. 9, 2010, pp. 1349-1353.

[10] Graham, W. R., Pitt Ford, C. W., and Babinsky, H., “An Impulse-Based Approach to Estimating Forces in Unsteady Flow,” Journal of Fluid Mechanics, Vol. 815, 2017, pp. 60-76.

[11] Corkery, S. J., and Babinsky, H., "Quantification of Added Mass using PIV Measurements for a Translating and Rotating Flat Plate," Journal of Fluid Mechanics, 2019.

[12] Gehlert, P., and Babinsky, H., "Linking the Unsteady Force Generation to Vorticity for a Translating and Rotating Cylinder," AIAA Scitech Proceedings, 2019, pp. 1-18.

[13] Corkery, S. J., and Babinsky, H., “An Investigation into the Added Mass Force for a Transverse Wing-Gust Encounter," AIAA Scitech 2019 Forum, 2019, pp. 1-18.

[14] Gehlert, P., and Babinsky, H., "Non-Circulatory Force Experienced by an Object of Finite Thickness Encountering a Sharp Edged Gust ( Invited ),", No. m, 2020, pp. 1-13.

[15] Didden, N., "On the Formation of Vortex Rings: Rolling-up and Production of Circulation," Journal of Applied Mathematics and Physics (ZAMP), Vol. 30, 1979.

[16] Sarpkaya, T., and Shoaff, R., "An Inviscid Model of Two Dimensional Vortex Shedding for Transient and Asymptotically Steady Separated Flow over a Cylinder,” Vol. 6, 2013.

[17] Xia, X., and Mohseni, K., "Unsteady Aerodynamics and Vortex-Sheet Formation of a Two-Dimensional Airfoil," Journal of Fluid Mechanics, Vol. 830, 2017, pp. 439-478.

[18] Goldstein, S., Modern Developments in Fluid Dynamics: an Account of Theory and Experiment Relating to Boundary Layers, Turbulent Motion and Wakes, Clarendon Press, 1938.

[19] Manar, F., Mancini, P., Mayo, D., and Jones, A. R., "Comparison of Rotating and Translating Wings: Force Production and Vortex Characteristics," AIAA Journal, Vol. 54, No. 2, 2015, pp. 519-530.

[20] Corkery, S. J., "Unsteady Aerodynamics of Wing Gust Encounters,” Ph.D. thesis, University of Cambridge, 2019.

[21] Corkery, S. J., Babinsky, H., and Harvey, J. K., "On the Development and Early Observations from a Towing Tank-Based Transverse Wing-Gust Encounter Test Rig,” Experiments in Fluids, Vol. 59, No. 9, 2018, pp. 1-16.

[22] Westerweel, J., “Fundamentals of Digital Particle Image Velocimetry,” Meas. Sci. Technol, Vol. 8, 1997, pp. 1379-1392.

[23] Raffel, M., Willert, C. E., and Kompenhans, J., Particle Image Velocimetry, $3^{\text {rd }}$ ed., Springer, 2018. 
[24] Wieneke, B., "PIV Uncertainty Quantification from Correlation Statistics," Measurement Science and Technology, Vol. 26, No. 7, 2015.

[25] Adrian, R. J., and Westerweel, J., Particle Image Velocimetry, Cambridge University Press, 2011.

[26] Prandtl, L., "Application of the "Magnus Effect" of the Wind Propulsion of Ships," National Advisory Commitee For Aeronautics, 1926.

[27] Tokumaru, P. T., and Dimotakis, P. E., "The Lift of a Cylinder Executing Rotary Motions in a Uniform low," Journal of Fluid Mechanics, Vol. 255, No. 1993, 1993, pp. 1-10. 